\title{
Diagnostic delay broadly associated with more advanced stage oral cancer
}

\author{
Abstracted from \\ Gomez I, Seoane J, Varela-Centelles P, Diz P, Takkouche B. \\ Is diagnostic delay related to advanced-stage oral cancer? A meta-analysis. \\ Eur J Oral Sci 2009; 117: 541-546 \\ Address for correspondence: Juan Seoane, Stomatology Department, \\ School of Medicine and Dentistry, University of Santiago de Compostela, \\ 15782 Santiago de Compostela, Spain. E-mail: juanmanuel.seoane@usc.es
}

\section{Question: Is diagnostic delay in oral cancer related to advanced stage disease at diagnosis?}

Data sources Medline, Embase, ISI Proceedings and the reference lists of relevant articles were used to find relevant studies.

Study selection Studies were included if: they presented original data from observational studies; included patients with a confirmed pathological diagnosis of oral or oropharyngeal squamous cell carcinoma; the outcome of interest was clearly defined as disease stage (TNM classification ); the exposure of interest was total diagnostic delay, defined as the period between the patient noticing either the first sign or symptom and definitive diagnosis (data were collected from interviews using a standardised questionnaire and medical records); provided relative risks (RR) and $95 \%$ confidence intervals $(\mathrm{Cl})$ or provided enough data to allow calculation of these figures.

Data extraction and synthesis Quality assessment was undertaken independently by two reviewers and followed the recommendations of the Meta-analysis of Observational Studies in Epidemiology (MOOSE). Meta-analysis was conducted using fixed and random-effects models. Results Nine studies carried out in nine different countries met the inclusion criteria and were included in the analysis. The fixed-effects pooled relative risk (RR) of advanced stages of oropharyngeal cancer when diagnostic delay is present was 1.32 (95\% Cl, 1.07-1.62). This association was stronger when the analysis was restricted to oral cancer (pooled RR, 1.47; 95\% Cl, 1.09-1.99) and when the delay was longer than 1 month (pooled RR, 1.69; 95\% Cl, 1.26-2.77).

Conclusions The probability for people with delayed diagnosis to present with an advanced-stage tumour at diagnosis was significantly higher than that of individuals with no delay in diagnosis. New prospective studies with strict methodology are needed, however, to shed more light on this association.

\section{Commentary}

The incidence of oral cancers continues to rise globally. Many of these cancers are diagnosed at advanced stages, resulting in extensive treatments and poor outcomes. Early diagnosis remains an important predictor of prognosis and survival and, therefore, this meta-analysis is very relevant.

The work investigated the association between diagnostic delay in oral cancer and advanced tumour stage at diagnosis. The review had clear inclusion criteria, the search included both English and nonEnglish language articles, and data were extracted independently by two clinicians and one epidemiologist. Nine retrospective crosssectional studies were included in the analysis.

Results showed that diagnostic delay is a risk factor for advanced stage oropharyngeal cancers. The association was stronger for oral cancer than for pharyngeal cancer. This is unsurprising, since pharyngeal cancers tend to metastasise early and may be diagnosed at late stages because of their anatomical location.

This meta-analysis contradicted a systematic review by Goy et al. ${ }^{1}$ The review found no evidence to support the relationship between diagnostic delay and tumour stage in head and neck cancers, including oral cancers. These counterintuitive findings, however, may have resulted from the high heterogeneity between studies in the review. Indeed, the results of the analysis here should be interpreted with caution because of the small number of studies included, their limited quality, and heterogeneity in their outcomes. In addition, the retrospective design of these studies may have introduced recall bias to the analysis. Moreover, the analysis did not account for tumour growth since this information was missing from included studies. This is particularly important since tumour growth rate was found to have a greater effect on diagnostic staging than diagnostic delay. ${ }^{2}$

Consequently, more research is required in this area. Future studies should aim to obtain more reliable data, avoid using clinical records as exclusive sources, and account for the variability of oral cancers resulting from factors such as tumour proliferation rate and cancer site. Furthermore, educational interventions are needed to promote early identification of oral cancer symptoms in patients and to increase health professionals' capacity to recognise malignant and premalignant lesions. Finally, in the absence of comprehensive screening programmes, opportunistic screening by care providers should be encouraged.

Imad AI-Dakkak

Centre for Evidence-based Dentistry, Oxford, UK

1. Goy J, Hall SF, Feldman-Stewart D, Groome PA. Diagnostic delay and disease stage in head and neck cancer: a systematic review. Laryngoscope 2009; 119: 889-898.

2. Symonds RP. Cancer biology may be more important than diagnostic delay. BrMed J 2002; 325: 774.

Evidence-Based Dentistry (2010) 11, 24. doi:10.1038/sj.ebd.6400708 